\title{
AVALIAÇÃO DA ESTIMULAÇÃO DE LPS NA MIGRAÇÃO CELULAR EM Rhamdia quelen EXPOSTOS À DELTAMETRINA
}

\author{
Evaluation of LPS Stimulant on Cell Migration of \\ Rhamdia quelen Exposed to Deltamethrin
}

\author{
Cláudia Turra Pimpão ${ }^{1}$ \\ Guilherme Borges Bond ${ }^{2}$ \\ Carine Budziak ${ }^{3}$ \\ Larissa Anuska Zeni Condas ${ }^{3}$ \\ Pedro Kaiseler ${ }^{3}$
}

\section{Resumo}

A deltametrina, pertencente ao grupo dos piretróides e amplamente empregada nas lavouras brasileiras, na medicina veterinária, no controle de pestes domésticas e na saúde pública, principalmente para controle de vetores, de modo geral os organismos não alvos, como as espécies aquáticas, são consideradas extremamente sensíveis aos piretróides. $\mathrm{O}$ objetivo deste estudo foi avaliar o efeito da dose subletal de deltametrina por parâmetros hematológicos e migração leucocitária no exsudato peritoneal em uma espécie de peixe fluvial nativo da região sul do Brasil, vulgarmente conhecido como Jundiá (Rhamdia quelen). Foram utilizados 4 grupos para análises sangüíneas (salina, LPS $100 \mu \mathrm{g} / \mathrm{kg}$, deltametrina $5 \mu \mathrm{g} / \mathrm{kg}$, LPS $100 \mathrm{mg} / \mathrm{kg}$ + deltametrina $5,0 \mathrm{mg} / \mathrm{kg}$, via IP), cada um contendo 10 peixes. As coletas de sangue e do líquido peritoneal foram realizadas 96 horas após a exposição ao inseticida e 4 horas após inoculação de LPS. A coleta de sangue foi feita por punção da veia caudal e após as amostras foram submetidas às contagens totais e diferenciais de leucócitos, contagens totais de eritrócitos, hematócrito e dosagem de hemoglobina. Para análise do exsudato peritoneal, foram utilizados 4 grupos contendo 10 peixes cada (salina, LPS $1 \mu \mathrm{g} / \mathrm{kg}$, deltametrina $5 \mathrm{mg} / \mathrm{kg}$ e deltametrina $5 \mathrm{mg} / \mathrm{kg}+\mathrm{LPS} 1 \mu \mathrm{g} / \mathrm{kg}$ ). Após a coleta, as amostras eram submetidas à contagem total de leucócitos. Para as análises estatísticas, foi utilizada análise de variância seguida por teste de Bonferroni para as comparações entre as médias. Os resultados obtidos revelaram que todos os grupos tratados apresentaram aumento significativo do número de leucócitos no sangue. A contagem total de eritrócitos variou significativamente no grupo exposto somente à deltametrina em relação aos demais grupos, sendo que os demais parâmetros analisados não foram significativos. Nas análises dos leucócitos do exsudato peritoneal, os grupos tratados mostraram diferença significativa em relação ao grupo salina. Os resultados sugerem que a subdose de deltametrina afeta o organismo do Jundiá, podendo causar policitemia, devido à compensação pela queda no volume corrente de oxigênio, podendo, também, ser considerada um agente inflamatónio para Rhamdia quelen, produzindo leucocitose tanto no sangue quanto na cavidade peritoneal.

Palavras-chave: Rhamdia quelen; LPS; Deltametrina; Migração celular.

1 Médica Veterinária, M.Sc. Professora de Farmacologia e Toxicologia Veterinária - PUCPR. Pontifícia Universidade Católica do Paraná. BR 376, km 14, Costeira, Cx. Postal 129, CEP 83010-500. São José dos Pinhais, Paraná, Brasil. claudia.pimpao@pucpr.br

2 Acadêmico do Curso de Medicina Veterinária - PUCPR / Bolsista do Programa PIBIC/CNPq

3 Acadêmicos do Curso de Medicina Veterinária PUCPR 


\section{Abstract}

Deltamethrin (DM) is a synthetic pyrethroid group insecticide widely applied in Brazilian crops, in the veterinary medicine, in the public health against vectors due to low toxicity to the man and other mammals. However, to not-target species such as the aquatic organisms, mainly the fishes, DM is extremely toxic. The aim of this study was to evaluate the effect of sub lethal doses of DM in hematological parameters and number of peritoneal leukocytes of Rhamdia quelen, a south Brazilian native river fish. For the blood experiment, four groups were used (control, LPS $100 \mu \mathrm{g} / \mathrm{kg}$, DM $5 \mathrm{mg} / \mathrm{kg}$ and LPS $100 \mu \mathrm{g} / \mathrm{kg}+\mathrm{DM} 5 \mathrm{mg} / \mathrm{kg}$, IP) and for the exudates peritoneal experiment, four groups were used too (control, LPS $1 \mu \mathrm{g} / \mathrm{kg}$, DM $5 \mathrm{mg} / \mathrm{kg}$ and $\mathrm{LPS} 1 \mu \mathrm{g} / \mathrm{kg}+\mathrm{DM} 5 \mathrm{mg} / \mathrm{kg}$, IP), each one with 10 fish. Fishes were exposed to DM or vehicle for $96 \mathrm{~h}$. After this period, each group received lipopolysaccharide (LPS, E.coli, 0111:B4) or saline. The results were analyzed by ANOVA followed by Bonferroni's test. DM and LPS increased erytrocyte number, and also increased the number of leukocytes in the blood compared to control animals. LPSinduced a significantleukocyte migration to the peritoneal cavity of the fish exposed to DM. After LPS stimulation, the leukocyte migration increased significantly. These results suggest that $\mathrm{DM}$ can be toxic to aquatic organisms. The immune system could be specially affected since the number of leukocytes in the blood and in the peritoneal cavity was increased. It shows an immune stimulation of the deltamethrin due the increase of the leukocyte migration in the place of the inflammation.

Keywords: Rhamdia quelen; LPS; Deltamethrin; Leukocytes migration.

\section{Introdução}

A preocupação com a conservação do ambiente é crescente, e com ela, as pesquisas em ecotoxicologia ganham maior importância. O desenvolvimento da indústria e o aumento desenfreado das populações levam à expansão das áreas agrícolas e, para suprir a demanda de alimentos, o uso de agrotóxicos torna-se mais freqüente. A utilização excessiva de um inseticida relativamente "seguro" pode levar ao descuido no manejo do produto e na proteção ambiental, podendo ocorrer a contaminação de ambientes aquáticos por estes pesticidas (OSWEILER, 1998).

A resposta leucocitária é uma ferramenta de diagnóstico muito utilizada na clínica veterinária, no entanto, a contagem das células brancas pode ser também usada como ferramenta clínica na medicina aquática, mas deve-se ter muito cuidado, pois existem vários fatores que limitam a interpretação. O fator biológico mais importante na acurácia das análises das células brancas é a variação em tipo, número e aparência dos leucócitos nas diferentes espécies de peixes. Os peixes apresentam os mesmos tipos celulares do que outros vertebrados, possuindo a mesma morfologia e a mesma função fisiológica do que os encontrados em mamíferos (BARTON; IWAMA, 1991).

O principal foco da hematologia de peixes tem sido: a determinação de efeitos das substâncias tóxicas e as mudanças nas condições am- bientais sobre as análises sangüíneas. Existem estudos bem documentados sobre as mudanças dos valores hematológicos, mas são correlacionados com situações de estresse (HRUBEC, et al. 1996).

Dentre os agrotóxicos, encontra-se a deltametrina, um piretróide de Classe II, que se apresenta na forma de cristais brancos e é utilizado como inseticida em lavouras, ectoparasiticidas de pequenos animais e bovinos e até no controle dos vetores da Malária (LARINI, 1999). Segundo Díaz-Barriga (2002), a deltametrina é particularmente tóxica para os peixes, apresentando as espécies, de modo geral, toxicidade aguda com concentrações muito baixas. Como relata Larini (1999), o quadro clínico de intoxicação por piretróides classe II parece ser de origem central, com salivação excessiva, movimentos irregulares dos membros, convulsões clônicas e tônicas e sensibilidade aumentada a estímulos externos.

O Rhamdia quelen ou Jundiá é uma espécie de peixe siluriforme de grande importância econômica no sul da América do Sul. Sua criação intensiva tem aumentado, o que faz com que esta espécie ganhe muito espaço no mercado consumidor (TAVARES-DIAS, 2002).

Este trabalho tem como objetivo analisar a influência da deltametrina sobre a resposta inflamatória do peixe teleósteo vulgarmente conhecido como Jundiá (Rhamdia quelen), oriundos de cativeiro, espécie nativa do sul da América do Sul. 


\section{Materiais e Métodos}

Os peixes utilizados nos experimentos são provenientes de cativeiro, coletados no Setor de Piscicultura do Patronato Santo Antônio/PUCPR. Após a coleta, eram transportados para o Laboratório de Farmacologia Veterinária da PUCPR, onde eram mantidos em aquários sob aeração e filtração e à temperatura constante de 18 a $20^{\circ} \mathrm{C}$ e pH 7,0. Os animais eram alimentados com ração comercial Ad libitum. Os peixes permaneceram por uma semana para aclimatação, antes das experimentações.

Para 0 experimento das análises do sangue, foram utilizados 4 grupos de 10 peixes cada: salina, $100 \mu \mathrm{g} / \mathrm{kg} \mathrm{LPS}, 5 \mathrm{mg} / \mathrm{kg}$ deltametrina, 100 $\mu \mathrm{g} / \mathrm{kg} \mathrm{LPS}+5,0 \mathrm{mg} / \mathrm{kg}$ deltametrina. As coletas sangǘneas foram realizadas 96 horas após exposição à deltametrina e 4 horas após inoculação de LPS (100 $\mu \mathrm{g} / \mathrm{kg}$ ). Para as contagens totais de leucócitos e enitrócitos e contagens diferenciais de leucócitos foram utilizadas metodologias de Tavares-Dias et al. $(1999 a, b ; 2000 a, b)$ e para avaliação de hemoglobina $(\mathrm{Hb})$ e hematócrito(Ht) a metodologia utilizada foi de acordo com Ellis (1977) e Hine (1992).
Na experimentação das análises do exsudato peritoneal, form utilizados 10 peixes por grupo: salina, $1 \mathrm{mg} / \mathrm{kg}$ LPS, deltametrina $5 \mathrm{mg} / \mathrm{kg}$ e deltametrina $5 \mathrm{mg} / \mathrm{kg}+1 \mathrm{mg} / \mathrm{kg}$ de LPS. Seguindo a metodologia de Pimpão et al. (2004a) e Afonso et al. (1997), os leucócitos foram coletados e contados.

Para as análises estatísticas, foi utilizada análise de variância, seguida por teste de Bonferroni para as comparações entre as médias.

\section{Resultados e discussão}

Após a realização do experimento piloto para determinar a dose subletal de Deltametrina, observou-se que a dose de $5,0 \mathrm{mg} / \mathrm{kg}$ provocava sinais de excitação do sistema nervoso típicos da intoxicação pelo piretróide nos peixes, não ocorrendo o óbito, portanto optou-se por esta dose.

De acordo com a Figura 1, podemos observar que os grupos tratados apresentaram uma elevação significativa no número de leucócitos em Rhamdia quelen em relação ao grupo salina. O grupo LPS (salina) também mostrou diferença significativa em relação aos grupos intoxicados com deltametrina.

FIGURA 1 - Número de leucócitos / $\mathrm{mm}^{3}$ de sangue em Rhamdia quelen. Figure 1 - Number of leukocytes / $\mathrm{mm}^{3}$ of blood in Rhamdia quelen.

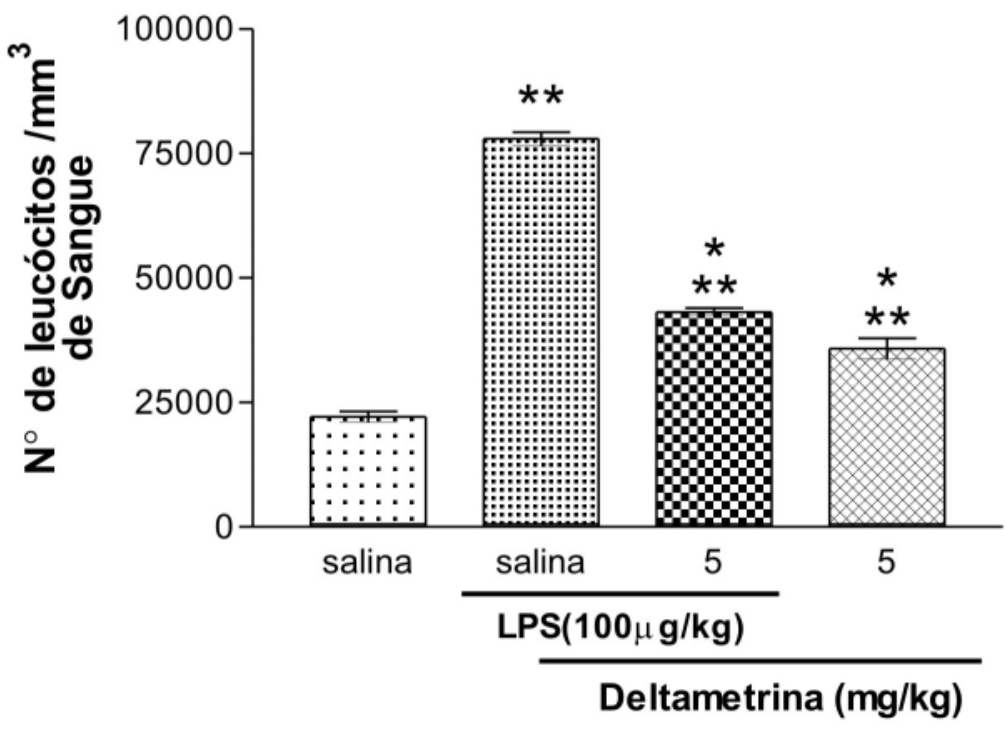

* Diferença em relação ao grupo salina $+\mathrm{LPS}, \mathrm{p}<0,001$.

** Diferença em relação ao grupo salina, $p<0,001$. 
A Figura 2 revela que a contagem total de eritrócitos variou significativamente no grupo ex- posto à deltametrina, havendo também diferença significativa do referido grupo com os grupos tratados com LPS.

FIGURA 2 - Número de eritrócitos / $\mathrm{mm}^{3}$ de sangue em Rhamdia quelen.

Figure 2 - Number of erythrocytes / mm3 of blood in Rhamdia quelen.

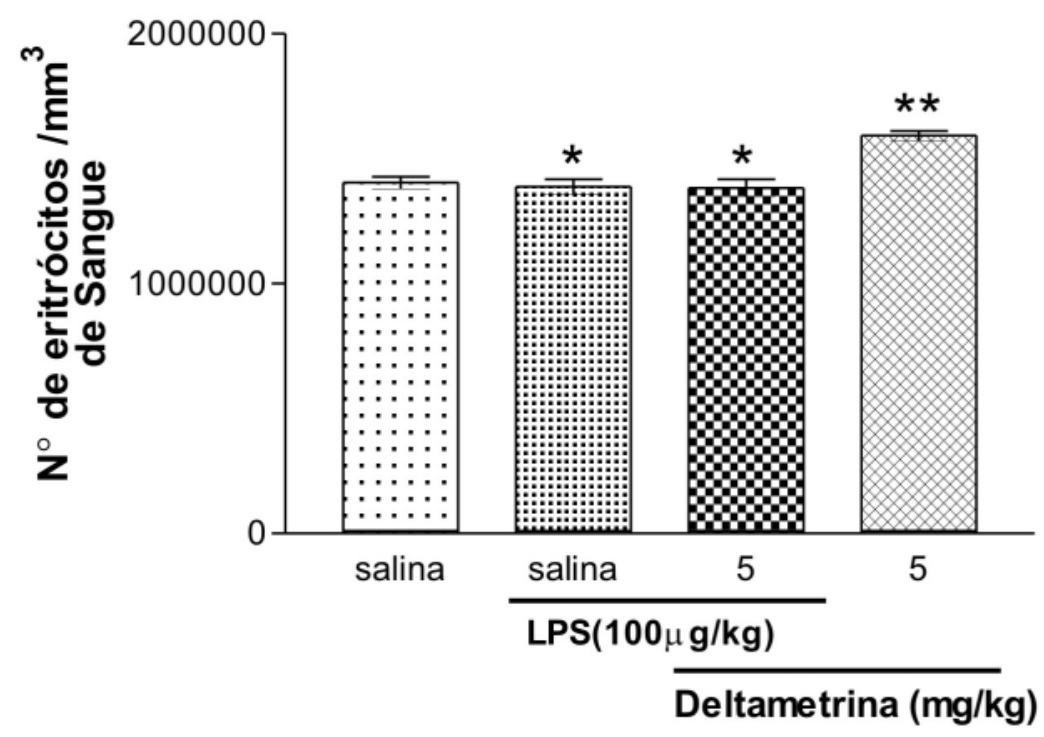

* Diferença em relação ao grupo salina, $\mathrm{p}<0,001$.

** Diferença em relação ao grupo deltametrina, $\mathrm{p}<0,001$.

De acordo com a Figura 3, podemos observar que existe uma população natural de leucócitos na cavidade peritoneal dos Jundiás e também que os grupos tratados mostraram diferença significativa em relação ao grupo salina, no entanto, o grupo LPS (salina) apresentou também nú- mero de leucócitos no exsudato peritoneal significativamente maior quando comparado aos grupos com deltametrina. Podemos observar também que o grupo deltametrina teve o número de leucócitos significativamente menor que o grupo deltametrina + LPS. 


\section{FIGURA 3 - Número de leucócitos / $\mathrm{mm}^{3}$ de exsudato peritoneal.}

Figure 3 - Number of leukocytes / $\mathrm{mm}^{3}$ of exudates peritoneal.

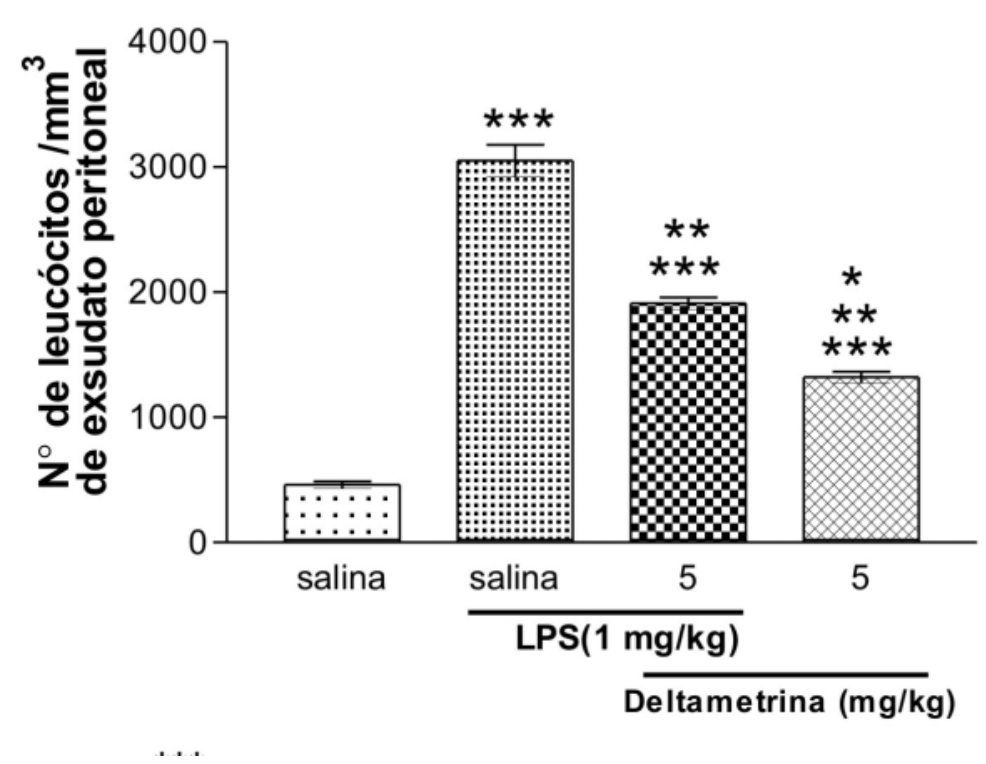

*** Diferença em relação ao grupo salina, $p<0,001$.

** Diferença em relação ao grupo salina $+\mathrm{LPS}, \mathrm{p}<0,001$.

* Diferença em relação ao grupo deltametrina +LPS, $p<0,001$.

\section{Conclusões}

Os valores médios para os parâmetros sanguíneos encontrados neste experimento são equivalentes aos encontrados na literatura para Rhamdia quelen: leucócitos do sangue 15502,40 \pm 22918,85 céls $/ \mathrm{mm}^{3}$; eritrócitos $1662500 \pm 223364$

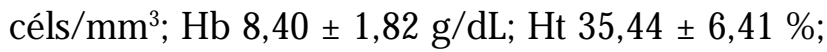
Volume Globular Médio 100,86 \pm 87,92 fl e Concentração de Hemoglobina Globular Média 27,87 $\pm 4,86 \mathrm{~g} / \mathrm{dL}$ (TAVARES DIAS; MORAES, 2004).

De acordo com os resultados obtidos, pudemos observar que o uso de LPS pode aumentar a contagem de leucócitos em Rhamdia quelen, incitando uma resposta inflamatónia, como foi observado por Pimpão et al. (2004b) utilizando cascudos. Da mesma forma, quando os peixes foram intoxicados com deltametrina, observou-se uma leucocitose, resultado também observado por Pimpão et al. (2004b), que concluíram que 0 referido inseticida pode atuar como agente inflamatório.
Outros inseticidas como organosfosforados produzem linfopenia em carpas (SVOBODA, 2001). Isso indica uma diminuição não específica da imunidade. Linfopenia como conseqüência de intoxicação aguda por parathion foi reportada por Nath; Banerjee (1996) e por Siwicki et al. (1990) em carpas após a intoxicação aguda por triclorfon. Também é sabido que os peixes podem apresentar leucopenia quando expostos ao DDT (IWAMA; NAKANISHI, 1996). Resultados semelhantes foram encontrados por Benarji; Rajendranath (1990) em relatos com intoxicação aguda por diclorvós em Clarias batrachus, por Tavares-Dias et al., 1999c, em triclorfon em Piaractus mesopotamus e em Oreochromis mossambicus. O número de eritrócitos dos peixes geralmente são mais baixos quando comparados com mamíferos, tendo uma taxa aproximadamente de 1 a $5 \times 10^{6} / \mu \mathrm{l}$ de sangue. A contagem de eritrócitos varia de acordo com a necessidade de oxigênio, segundo Hrubec et al. (1996). 
O número de eritrócitos dos Rhamdia quelen que foram expostos à deltametrina aumentaram significativamente, podendo ser explicado devido ao mecanismo de ação da deltametrina que age prolongando a abertura dos canais de sódio, provocando despolarização da membrana, como também podendo inibir a ação do gaba, portanto estimulando o Sistema Nervoso Central e levando à hipóxia, ou seja, anóxia de tecidos periféricos (CASIDA et al., 1983).

Segundo Rifkind et al. (1980), o aumento do número de eritrócitos vem sendo mostrado em estudos laboratoriais em diversas espécies quando expostas à hipóxia crônica, nestas condições parece que o baço e talvez o fígado possam reativar a eritropoese para compensar a demanda pelo aumento do transporte de oxigênio para os tecidos periféricos. Índices de hemoglobina e eritrócitos têm sido determinados em vários estudos, mas não há resultados conclusivos que possam ser relacionados com problemas de anemias. Contudo, pode haver, ainda, diferenças na metodologia de colheita de sangue, como tipo de anticoagulante e via de administração utilizada, as quais também podem atuar como fonte de variação de resultados hematológicos em peixes. Outros fatores, como estado nutricional, sazonalidade, maturação gonadal, sexo e variação genética também podem influenciar significativamente as variáveis hematológicas, fatores estes também observados neste estudo (KORISIAKPERE, 1985).

Na avaliação do exsudato peritoneal verificou-se que existe naturalmente uma população leucocitária na cavidade peritoneal do Rhamdia quelen e quando se inocularam doses pequenas de LPS, houve uma migração leucocitária significativa no local inoculado. Também ocorreu leucocitose no local inoculado nos peixes expostos à deltametrina, o que significa que este piretróide pode também provocar uma reação inflamatória local. Resultados semelhantes foram encontrados em cascudos expostos à deltametrina por Pimpão et al. (2004a).

Os valores normais de leucócitos, eritrócitos, taxa de hemoglobina e hematócrito encontrados são equivalentes aos valores encontrados na literatura. Também podemos considerar que os valores hematológicos podem ser mais elevados do que o normal quando esta espécie sofrer exposição pelo LPS e pela deltametrina, pois se conclui que estes valores sofrem elevação quando o organismo é invadido por um microorganismo e que a deltametrina por si só produz leucocitose no sangue, podendo ser considerado também um agente inflamatório para Rhamdia quelen.

Podemos concluir que existe naturalmente uma população leucocitária na cavidade peritoneal do Rhamdia quelen; quando se inoculam doses pequenas de LPS, ocorre migração leucocitária significativa no local inoculado, neste caso na cavidade peritoneal, sendo que a deltametrina também pode ser considerada um agente inflamatório. E quando ocorrer a exposição do Rhamdia quelen à deltametrina e já houver um foco inflamatório, os danos ao organismo podem ser agravados.

\section{Referências}

AFONSO, A.; ELUIS, A. E.; SILVA, M. T. The leukocyte population of the unstimulated peritoneal cavity of the rainbow trout (Oncorhynchus mykiss). Fish \& Shellfish Immunology, v. 7, p. 335- 348, 1997.

BARTON, B. A.; IWAMA, G. K. Physiological changes in fish from stress in aquaculture with emphasis on the response and effects of corticosteroids. Ann. Rev. Fish Dis. v.1, p. 3-26, 1991.

BENARJI, G.; RAJENDRANATH, T. Haematological changes induced by an organophosphorus insecticide in a freshwater fish Clarias batrachus (Linnaeus). Trop. Freswat. Biol. v. 2, p. 197-202, 1990.

CASIDA, J. E.; GAMMON, D. W.; GLICKMAN, A. H. Mechanisms of selective action of pyrethroid insecticides. Annu. Rev. Pharmacol. Toxicol. v. 23, p. 413-438, 1983.

DIAZ-BARRIGA, F. Factores de Exposición y Toxicidad Del DDT y de la Deltametrina en Humanos y en Vida Silvestre. Informe Técnico da Facultad de Medicina, Universidad Autónoma de San Luis de Potosí, México, 2002

ELUS, A. E. The leukocytes of fish: a review. Journal of Fish Biology, v.11, p. 453 - 491, 1977.

HINE, P. M. The granulocytes of fish. Fish \& Shellfish Immunology, v. 2, p. 79 - 98, 1992. 
HRUBEC, T. C. et al. Comparasion of hematologic reference intervals between culture system and type of hybrid striped bass. Am. J. Vet. Res. v.57, p. 618 - 623, 1996.

IWAMA, G., NAKANISHI, T. The Fish Immune System, Organism, Pathogen and Environment. Academic Press. San Diego, Califórnia,1996. v. 15.

KORI-SIAKPERE, O. Haematological characteristics of Clarias isheriensis Sydenham. J. Fish. Biol. 27, p. 259 - 263, 1985.

LARINI, L. Toxicologia dos praguicidas. São Paulo,SP: Manole, 1999.

NATH, R; BANERJEE, V.(1996). Effect of pesticides methylparathion and cypermethrin on the airbreathing fish Heteropneustes fossilis. Environ. Ecol. 14, p. 163-165, 1996.

OSWEILER, G. D. Toxicologia Veterinária. Porto Alegre: Artes Médicas, 1998.

PIMPÃO, C. T.; ZAMPRONIO, A. R. SILVA DE ASSIS, H. C. 2004. Leukocyte migration to the peritoneal cavity in Ancistrus multispinis (Fish, Teleostei) exposed to Deltamethrin. In: CONGRESSO BRASILEIRO DE FARMACOLOGIA ETERAPÊUTICA EXPERIMENTAL, 36., Anais... 2004a.

PIMPÃO, C. T.; ZAMPRONIO, A. R.; SILVA DE ASSIS, H. C. 2004. Effect of Deltamethrin in haematological parameter and number of resident peritonelal leukocytes of Ancistrusmultispinis(Fish, Teleostei). In: CONGRESSO BRASILEIRO DE FARMACOLOGIA E TERAPÊUTICA EXPERIMENTAL, 36., Anais... 2004b.

RIFKIND, R. A. et al. Fundamentals of Hematology, 2. ed. Chicago:Yearbook Medical, 1980.

SVOBODA, M. et al. Leucocytes profiles of diploid and triploid tench, Tinca tinca L Aquaculture, v.198, p. 159 - 168, 2001.

SIWICKI, A. K. et al. In vivo effect of the organophosphorus insecticide trichlorphon on imunne response of carp (Cyprinus carpio). II. Effect of high doses of trichlorphon on nonspecific immune response. Ecotox. Environ. af. v.19, p. 99-105, 1990.
TAVARES - DIAS, M. et al. Características Hematológicas de Teleósteos Brasileiros II. Parâmetros sangüíneos do Piaractusmesopotamicus Holmberg, 1887 (Osteichthyes:Characidae) em policultivo intensivo. Ver. Bras. Zool. Curitiba, v.16, p. 423 - 431, 1999a.

TAVARES - DIAS, M. et al. Características Hematológicas de Teleósteos Brasileiros IV. Parâmetros eritroleucométricos, trombométricos e glicemia do matrinxã Brycon cephalus Günther, 1869 (Osteichthyes:Characidae). Ars. Veterinária, Jaboticabal, v.15, p. 149 - 153, 1999 b.

TAVARES - DIAS, M.; MARTINS M. L.; KRONKA, S. $\mathrm{N}$. Evaluation of the haematological parameters in Piaractusmesopotamicus Holmberg (Osteichthyes: Characidae) with Argulussp. (Crustacea, Brachiura) infestation and treatment with organophosphate. Revta. Bras. Zool. v.16, p. 553 - 555, 1999c.

TAVARES - DIAS, M. et al. Haematological Characteristics of Brazilian Teleosts III. Parameters of the hybrid tambacu (Piaractusmesopotamicus $\mathrm{x}$ Colossoma macropomum) (O steichthyes:Characidae). Rev. Bras. Zool. Curitiba, v.17, p. 899 - 926, 2000a.

TAVARES - DIAS, M. et al. Haematological characteristics of hybrid Florida red tilapia, Oerochromis urolepis horn orun x O. mossambicus underintensive rearing. In: International Simposium on Tilapia Aquaculture. Proccedings... Rio de Janeiro, Rio de Janeiro: Fitzmmons, K., Carvalho Fillhos, J. 2000b. p. 533 - 541.

TAVARES- DIAS, M. Características Hematológicas de Teleósteos Brasileiros. IV. Variáveis do Jundiá Rhamdia quelen (pimelodidae). Rev. Ciência Rural, v. 32, n. 4, p. 693-698, jul./ago. 2002

TAVARES - DIAS, M.; MORAES F. R. Hematologia de Peixes Teleósteos. São Paulo,SP 2004.

Recebido em: 29/06/2005 Aprovado em: 30/09/2005 